\title{
Intracranial pressure waveform characteristics in idiopathic normal pressure hydrocephalus and late-onset idiopathic aqueductal stenosis
}

\author{
Lauren M. Green ${ }^{1}$, Thomas Wallis ${ }^{1}$, Martin U. Schuhmann ${ }^{2}$ and Matthias Jaeger ${ }^{1,3,4,5^{*}}$ (1)
}

\begin{abstract}
Background: Idiopathic normal pressure hydrocephalus (iNPH) and late-onset idiopathic aqueductal stenosis (LIAS) are two forms of chronic adult hydrocephalus of different aetiology. We analysed overnight intracranial pressure (ICP) monitoring to elucidate ICP waveform changes characteristic for iNPH and LIAS to better understand pathophysiological processes of both diseases.

Methods: 98 patients with iNPH and 14 patients with LIAS from two neurosurgical centres were included. All patients underwent diagnostic overnight computerised ICP monitoring with calculation of mean ICP, ICP heartbeat related pulse wave amplitude calculated in the frequency domain (AMP) and the time domain (MWA), index of cerebrospinal compensatory reserve (RAP) and power of slow vasogenic waves (SLOW).

Results: ICP was higher in LIAS than iNPH patients $(9.3 \pm 3.0 \mathrm{mmHg}$ versus $5.4 \pm 4.2 \mathrm{mmHg}, \mathrm{p}=0.001)$. AMP and MWA were higher in iNPH versus LIAS $(2.36 \pm 0.91 \mathrm{mmHg}$ versus $1.81 \pm 0.59 \mathrm{mmHg}$ for AMP, $p=0.012 ; 6.0 \pm 2.0$ $\mathrm{mmHg}$ versus $4.9 \pm 1.2 \mathrm{mmHg}$ for MWA, $p=0.049)$. RAP and SLOW indicated impaired reserve capacity and compliance in both diseases, but did not differ between groups. INPH patients were older than LIAS patients (77 \pm 6 years versus $54 \pm 14$ years, $p<0.001$ ).

Conclusions: ICP is higher in LIAS than in INPH patients, likely due to the chronically obstructed CSF flow through the aqueduct, but still in a range considered normal. Interestingly, AMP/MWA was higher in iNPH patients, suggesting a possible role of high ICP pulse pressure amplitudes in iNPH pathophysiology. Cerebrospinal reserve capacity and intracranial compliance is impaired in both groups and the pressure-volume relationship might be shifted towards lower ICP values in iNPH. The physiological influence of age on ICP and AMP/MWA requires further research.
\end{abstract}

Keywords: Idiopathic normal pressure hydrocephalus, Aqueductal stenosis, Intracranial pressure, Neuromonitoring, Intracranial compliance, Intracranial pulsations

\section{Background}

Idiopathic normal pressure hydrocephalus (iNPH) is part of the large group of neurodegenerative diseases

*Correspondence: m.jaeger@unsw.edu.au

1 Department of Neurosurgery, Wollongong Hospital, Loftus Street, Wollongong, NSW 2500, Australia

Full list of author information is available at the end of the article affecting the aging population. It is characterised by a clinical triad of progressive gait impairment, decline in cognitive function and incontinence. The natural history of iNPH is generally unfavourable with worsening neurological symptoms and reduced survival $[1,2]$. Treatment of iNPH is surgical insertion of a ventriculo-peritoneal shunt (VP-shunt), which can lead to improved symptoms

(c) The Author(s) 2021. This article is licensed under a Creative Commons Attribution 4.0 International License, which permits use, sharing, adaptation, distribution and reproduction in any medium or format, as long as you give appropriate credit to the original author(s) and the source, provide a link to the Creative Commons licence, and indicate if changes were made. The images or other third party material in this article are included in the article's Creative Commons licence, unless indicated otherwise in a credit line to the material. If material is not included in the article's Creative Commons licence and your intended use is not permitted by statutory regulation or exceeds the permitted use, you will need to obtain permission directly from the copyright holder. To view a copy of this licence, visit http://creativeco mmons.org/licenses/by/4.0/. The Creative Commons Public Domain Dedication waiver (http://creativecommons.org/publicdomain/ zero/1.0/) applies to the data made available in this article, unless otherwise stated in a credit line to the data. 
in selected patients and makes iNPH one of the few potentially treatable forms of dementia.

The pathophysiological processes of iNPH remain incompletely understood, resulting in a lack of clear diagnostic criteria. This makes selection of patients for surgical treatment and differentiation from other neurodegenerative diseases a challenge. Current evidence indicates that $\mathrm{iNPH}$ is thus underdiagnosed and undertreated [3]. It is now well accepted that iNPH is not only an imbalance of cerebrospinal fluid (CSF) production and reabsorption, but represents a more complex disturbance of CSF dynamics that becomes symptomatic at the late stages of life. There is therefore a need for better understanding of the pathophysiology of iNPH [4].

Late-onset idiopathic aqueductal stenosis of the adult (LIAS) is a less common form of chronic adult hydrocephalus [5]. The underlying pathology of LIAS is thought to be chronically impaired CSF flow through a narrowed aqueduct, typically leading to a well defined radiological entity with enlarged lateral and third ventricles (triventricular hydrocephalus), together with a convex tuber cinereum and lamina terminalis and dilatation of the third ventricular recesses, thought to arise from a chronic CSF accumulation and intracranial pressure (ICP) elevation "upstream" from the aqueduct. The aqueductal pathology is likely present for decades, possibly since childhood, and patients become symptomatic after years of compensated ventriculomegaly. The clinical presentation is very similar to iNPH with gait impairment, cognitive decline and incontinence, but the age at presentation is mostly younger. Headaches can also be part of the clinical presentation and are thought to arise from the chronic supratentorial ICP elevations.

The aim of this study was to investigate differences between the physiologic ICP waveform characteristics of patients with iNPH and LIAS. Despite the similar clinical features of these two forms of chronic adult hydrocephalus, we hypothesised that the relatively well-defined entity of LIAS would present with a distinct pattern of ICP features attributable to the impaired aqueductal CSF flow. Differentiation of the LIAS ICP-profile from the ICP-profile of iNPH might help to elucidate ICP characteristics for iNPH and LIAS and thus provide insight into pathophysiological processes of both diseases.

\section{Methods}

We included patients treated at the Department of Neurosurgery, Wollongong Hospital, Illawarra Shoalhaven Local Health District, NSW, Australia between 20132020 and the Department of Neurosurgery, Eberhard Karls University Hospital, Tübingen, Germany, between 2009-2018 under the care of the two senior authors. Included patients underwent diagnostic intracranial pressure monitoring for investigation of iNPH or LIAS and were retrospectively identified from the ICP monitoring databases at both institutions. Clinical records, MRI and CT scans of the brain were reviewed for all patients.

\section{Patient characteristics, definition of iNPH and LIAS}

The patient selection process and inclusion procedure is described in detail in Fig. 1. Patients with iNPH had probable or possible iNPH as described in the iNPHguidelines [6], meaning they had ventricular enlargement on MRI and CT brain imaging without evidence of aqueductal stenosis or other CSF flow obstruction, together with appropriate clinical findings and no history of other causes of secondary NPH, such as brain haemorrhage or meningitis.

LIAS patients had chronic symptoms as described by Fukuhara et al. with more than 6 months duration [5]. They had radiological evidence on MRI of aqueductal stenosis with a normal sized fourth ventricle, dilatation of the lateral and third ventricles with a convex tuber cinereum and lamina terminalis and dilatation of the third ventricular recesses, meaning downward bulging of the floor of the third ventricle and forward bulging of the lamina terminalis (Fig. 2). Patients with presentation of aqueductal stenosis with clinical signs and symptoms of elevated ICP were not part of this investigation as both

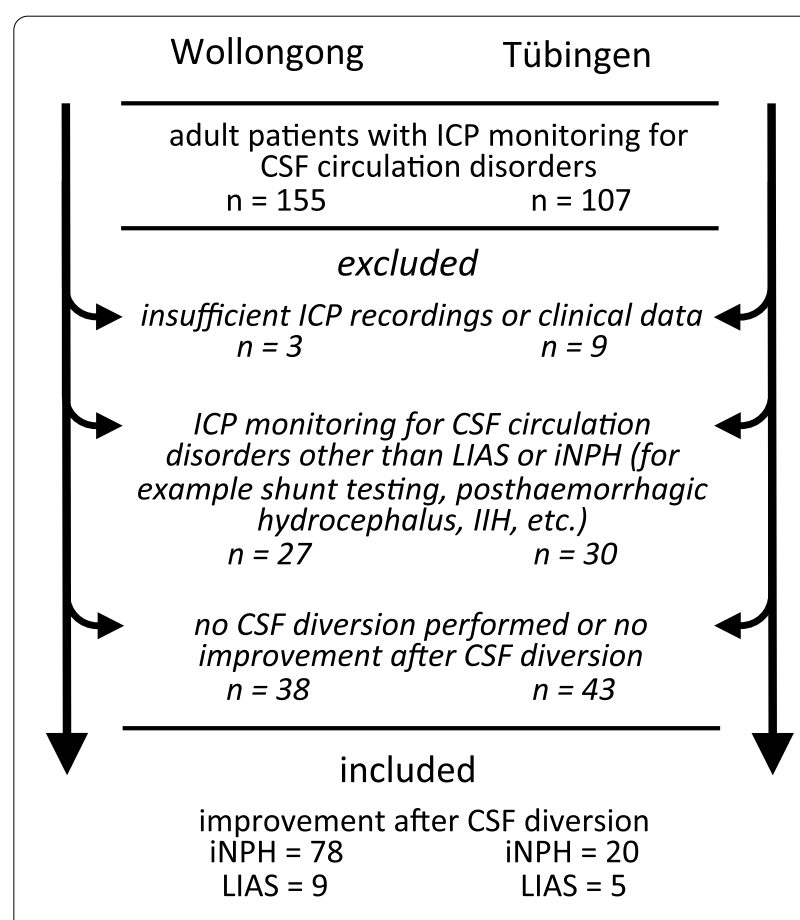

Fig. 1 Selection process for the study population. Adult patient were identified from the ICP monitoring database at both institutions 
study centres would typically not see the need for diagnostic ICP monitoring.

We only included patients with neurological improvement after CSF diversion in the final analysis. This is because we see improvement post shunting as the diagnostic gold standard and confirmation that patients suffered from symptomatic hydrocephalus and not other neurological disease. The disease severity of the iNPH patients was assigned retrospectively after review of the patients' clinical notes using the Oslo-iNPH grading scale [7]. This simple scale allocates $1-5$ points according to disease severity to each of the three domains of iNPH, gait impairment, cognitive impairment and incontinence. Patients receive a score of $3-15$ points, with lower scores representing a more unfavourable clinical status. No specific scale to quantify disease severity exists for LIAS and we used simple symptom description for these patients. The indication for CSF diversion via VP-Shunt or endoscopic third ventriculostomy (ETV) was made by the treating teams after review of the individual clinical and radiological information, together with ICP data and CSF infusion studies. Outcome was assessed using the OsloiNPH scale from the clinical notes at the 1-3 month postoperative follow-up appointment. The Evans index to describe the degree of ventricular dilatation was recorded for each patient from diagnostic MRI or CT brain [8].

\section{ICP monitoring and data recording}

Patients underwent diagnostic ICP monitoring for evaluation of chronic hydrocephalus as part of routine management. An ICP transducer was inserted through a right frontal burr hole. At Wollongong Hospital a Codman microsensor ICP transducer (Codman \& Shurtleff, Raynham, MA, USA) was used, in Tübingen a Neurovent P sensor (Raumedic AG, Helmbrechts, Germany). ICP

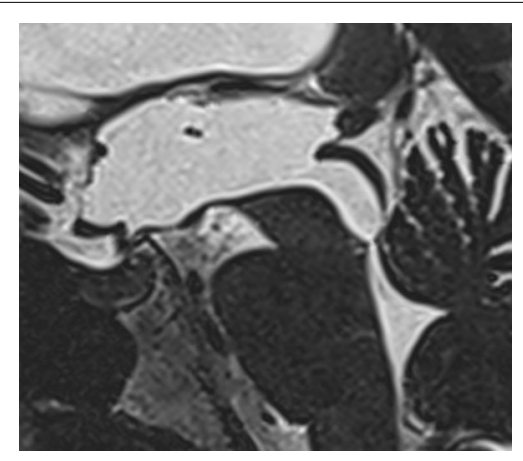

Fig. 2 Sagittal T2 MRI image through the third ventricle, cerebral aqueduct and fourth ventricle showing typical findings for LIAS. Note the convex shape of the tuber cinereum and lamina terminalis, dilated third ventricle, stenosis of the distal aqueduct and normal sized fourth ventricle monitoring was performed on a neurosurgical ward for 1-3 nights. The ICP raw data signal was digitally sampled using ICM + software (Cambridge University, Cambridge, UK) [9]. During the study period the raw data sampling frequency increased from 50 to $200 \mathrm{~Hz}$ with 15 patients recorded at $50 \mathrm{~Hz}, 20$ patients at $100 \mathrm{~Hz}$ and 77 patients at $200 \mathrm{~Hz}$. To minimise artefacts in the ICP signal only overnight data during the patients' sleep from a $10 \mathrm{~h}$ period from about 21:00 to 07:00 was analysed, typically from the first night a full $10 \mathrm{~h}$ recording was available. Artefacts, such as temporary disconnection of the ICP monitor, were manually eliminated after visual inspection of the ICP raw data.

\section{ICP waveform parameters}

The ICP raw data was analysed using ICM + software version 8.3 for the following mean parameters from the 10-hour overnight monitoring:

1. The mean ICP was calculated.

2. The ICP pulse pressure amplitude (AMP): AMP was calculated from the amplitude of the fundamental harmonic in the frequency range of 30-140 beats per min (bpm) over $10 \mathrm{~s}$ intervals and averaged every $60 \mathrm{~s}$.

3. Index of cerebrospinal compensatory reserve capacity (RAP): This is a marker of cerebrospinal compensatory reserve capacity and indirectly of compliance $[10,11]$. It is calculated as the moving Pearson correlation coefficient between 30 consecutive samples of ICP and AMP averaged over $10 \mathrm{~s}$. This $5 \mathrm{~min}$ time window was updated every $60 \mathrm{~s}$. RAP just above zero indicates normal reserve capacity and compliance when the physiological slow wave fluctuations in ICP do not induce significant changes in AMP. RAP $>0.6$ suggests impaired compliance when AMP correlates with slow wave ICP oscillations.

4. Heart rate (HR): HR was calculated from the frequency of the fundamental harmonic in the frequency range of $30-140 \mathrm{bpm}$ over $10 \mathrm{~s}$ intervals and averaged every $60 \mathrm{~s}$.

5. The ICP amplitude of the respiration induced waves (RESP): RESP was calculated from the amplitude of the fundamental harmonic in the frequency range of $7-30 \mathrm{bpm}$ over a $120 \mathrm{~s}$ time window and updated every $60 \mathrm{~s}$.

6. Respiratory rate (RR): RR was calculated from the frequency of the fundamental harmonic in the frequency range of 7-30 bpm over a $120 \mathrm{~s}$ time window and updated every $60 \mathrm{~s}$. 
7. The amplitude of the slow vasogenic waves of the ICP signal (SLOW): SLOW was calculated from the square root of the power in the frequency range of $0.3-3 \mathrm{bpm}$ over a 10 min time window updated every $60 \mathrm{~s}$ [12].

The ICP raw data was also analysed in the time domain using Sensometrics software (Sensometrics 4.0.2.4, dPCom A/S, Oslo, Norway) [13]. The following parameters were calculated:

8. The ICP mean pulse pressure wave amplitude in the time domain (MWA): The software algorithm identifies the amplitude of each single heartbeat induced pressure wave in the continuous ICP signal (the difference between diastolic minimum and systolic maximum pressure). MWA is calculated as the mean ICP pulse pressure wave amplitude for consecutive $6 \mathrm{~s}$ time windows. The software algorithm also identifies artefacts and time windows containing less than four valid heartbeat induced ICP waves are excluded.

9. Rise time of the ICP pulse pressure wave (RT): The RT was calculated as the time from the diastolic minimum to the systolic maximum for each heartbeat related ICP wave for consecutive $6 \mathrm{~s}$ time windows.

10. Rise time coefficient of the ICP pulse pressure wave (RTc): The RTc was calculated as the ratio of the amplitude over the rise time of each heartbeat induced ICP wave for consecutive $6 \mathrm{~s}$ time windows.

\section{Statistical analysis}

Prism software (Version 8.4.2, GraphPad LLC, San Diego, CA, USA) was used for statistical analysis. We used the Mann-Whitney-U test and chi-square test for comparison of variables between groups. Multiple linear regression models were used to analyse for potential confounders on the dependent variables. Correlation between selected parameters was calculated using nonparametric Spearman correlation coefficient. The probability of a type- 1 error $(\alpha)$ of $5 \%$ was accepted as being statistically significant.

\section{Results}

98 patients with iNPH showing improvement after VP-shunt insertion were included in this study. The median preoperative Oslo-score was 11 (range 4-14) and the median postoperative Oslo-score was 13 (range 7-15). They showed a median of 2 points (range 1-8) of improvement after shunt surgery. 14 patients with LIAS showing neurological improvement after VPShunt $(n=9)$ or ETV $(n=5)$ were included. 10 patients had improvement in gait, 8 in cognitive function, 7 in incontinence, and 7 reported better headache control. 11 patients had improvement in more than one domain. LIAS patients were younger and had a higher Evans index. Demographic and radiological data is summarised in Table 1.

\section{ICP waveform characteristics}

Figure 3 shows an individual example of $10 \mathrm{~h}$ overnight ICP monitoring and the waveform derived parameters AMP, RAP, RESP and SLOW.

Table 2 shows the ICP waveform characteristics between the iNPH and LIAS groups. Mean ICP was higher in LIAS, but still in a range considered normal (Fig. 4). Mean AMP and MWA were higher in iNPH (Fig. 5). RT was higher in iNPH, but RTc was similar (Fig. 6). Of note, all these statistically different parameters displayed overlap in individual ICP and derived values between groups. Other ICP derived parameters were not statistically different between groups. Due to technical difficulties the ICP raw data from $2 \mathrm{iNPH}$ patients from Tübingen, recorded at $50 \mathrm{~Hz}$, could not be analysed with Sensometrics software and thus analysis of MWA, RT and RTc in the iNPH group is from 96 patients only.

We found a significant correlation between ICP pulse wave amplitude calculated in the frequency domain (AMP) and time domain (MWA) of $\mathrm{r}=0.93, \mathrm{p}<0.001$.

In a multiple linear regression model age had a confounding effect on ICP between disease groups (iNPH versus LIAS) ( $p=0.001$, adjusted $\left.R^{2}=0.15\right)$. In another multiple linear regression model age had no confounding effect on AMP $\left(p=0.19\right.$, adjusted $\left.R^{2}=0.04\right)$. Both models were significant, but had a low adjusted $\mathrm{R}^{2}$.

\section{Differences between the two study centres}

In patients with iNPH ICP was higher in Tübingen than Wollongong in the overnight monitoring $(9.0 \pm 3.8$ mmHg versus $4.5 \pm 3.8 \mathrm{mmHg}, \mathrm{p}<0.001)$. RR was higher in Tübingen than in Wollongong $(15.5 \pm 2.9 \mathrm{bpm}$ versus

Table 1 Demographic and radiological details of iNPH and LIAS groups

\begin{tabular}{|c|c|c|c|}
\hline Variable & iNPH & LIAS & $p$ value \\
\hline No. of patients & 98 & 14 & \\
\hline Age (years) & $76.6 \pm 5.6$ & $53.8 \pm 14.4$ & $<0.001$ \\
\hline Female/male & $33 / 65$ & $8 / 6$ & 0.09 \\
\hline Evans-index & $0.39 \pm 0.04$ & $0.41 \pm 0.05$ & 0.03 \\
\hline $\begin{array}{l}\text { Duration of artefact free ICP data } \\
\text { obtained during } 10 \text { h of overnight } \\
\text { monitoring (min) }\end{array}$ & $593 \pm 16$ & $583 \pm 39$ & 0.67 \\
\hline
\end{tabular}

Given values are the mean \pm standard deviations or absolute numbers. $p$ values from Mann-Whitney-U test and chi-square test 


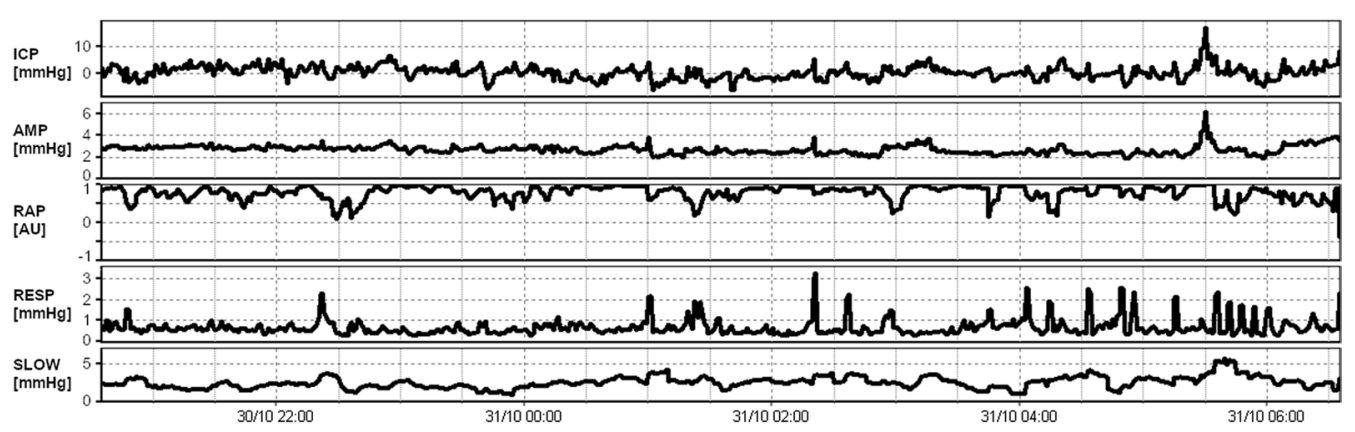

Fig. 3 Typical 10 h overnight recording of ICP, AMP, RAP, RESP and SLOW

Table 2 ICP derived parameters between iNPH and LIAS

\begin{tabular}{llll}
\hline Variable & iNPH & LIAS & p value \\
\hline No. of patients & 98 & 14 & \\
ICP $(\mathrm{mmHg})$ & $5.4 \pm 4.2$ & $9.3 \pm 3.0$ & 0.001 \\
AMP $(\mathrm{mmHg})$ & $2.36 \pm 0.91$ & $1.81 \pm 0.59$ & 0.012 \\
MWA $(\mathrm{mmHg})$ & $6.0 \pm 2.0$ & $4.9 \pm 1.2$ & 0.049 \\
RAP $(\mathrm{AU})$ & $0.67 \pm 0.17$ & $0.66 \pm 0.11$ & 0.59 \\
HR $(1 / \mathrm{min})$ & $69 \pm 10$ & $69 \pm 11$ & 0.88 \\
RESP $(\mathrm{mmHg})$ & $0.52 \pm 0.20$ & $0.66 \pm 0.59$ & 0.69 \\
RR $(1 / \mathrm{min})$ & $14 \pm 3$ & $14 \pm 2$ & 0.64 \\
SLOW $(\mathrm{mmHg})$ & $1.63 \pm 0.49$ & $1.78 \pm 0.72$ & 0.59 \\
RT $(\mathrm{s})$ & $0.25 \pm 0.04$ & $0.21 \pm 0.06$ & 0.014 \\
RTC $(\mathrm{mmHg} / \mathrm{s})$ & $25.2 \pm 9.9$ & $27.5 \pm 12.1$ & 0.61 \\
\hline
\end{tabular}

Given values are the mean \pm standard deviations. p values from MannWhitney-U test

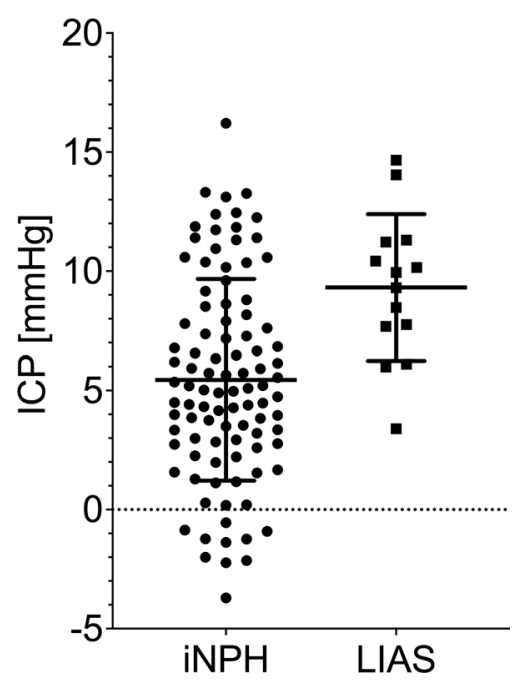

Fig. 4 Values of ICP for iNPH and LIAS. Horizontal bars are the mean \pm standard deviation. The difference between the groups was statistically significant $(p=0.001)$

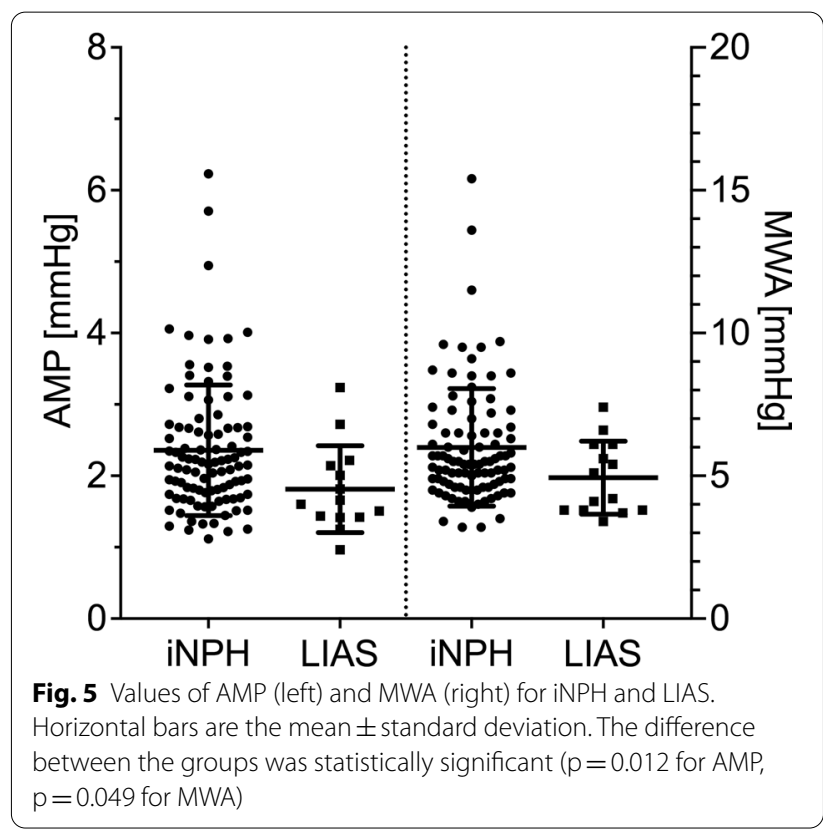

$13.9 \pm 2.5 \mathrm{bpm}, \mathrm{p}=0.024)$. AMP did not differ between centres in iNPH patients $(2.1 \pm 0.7 \mathrm{mmHg}$ versus $2.4 \pm 0.9 \mathrm{mmHg}, \mathrm{p}=0.25$, for Tübingen and Wollongong, respectively). MWA was also not different between centres in iNPH $(5.6 \pm 1.6 \mathrm{mmHg}$ versus $6.1 \pm 2.1 \mathrm{mmHg}$, $\mathrm{p}=0.52$, for Tübingen and Wollongong, respectively). Other clinical and monitoring parameters did not differ between centres in iNPH patients.

In LIAS patients the Evans index was lower in the Tübingen cohort $(0.36 \pm 0.05$ versus $0.44 \pm 0.02$, $\mathrm{p}=0.02$ ). From the ICP derived parameters the following showed statistically different values between Tübingen and Wollongong, respectively: RR, $13.0 \pm 1.1 \mathrm{bpm}$ versus $15.1 \pm 2.0 \mathrm{bpm}, \mathrm{p}=0.04$; RESP, $0.34 \pm 0.07 \mathrm{mmHg}$ versus $0.84 \pm 0.68 \mathrm{mmHg}, \mathrm{p}=0.012$; and $\mathrm{RTc}, 19.7 \pm 5.0$ $\mathrm{mmHg} / \mathrm{s}$ versus $31.9 \pm 12.7 \mathrm{mmHg} / \mathrm{s}, \mathrm{p}=0.019)$. Other 


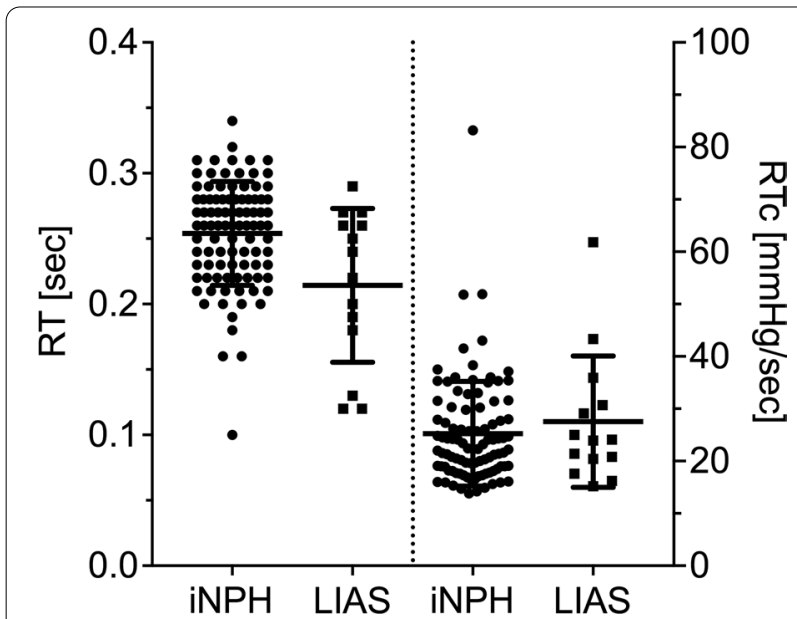

Fig. 6 Values for RT (left) and RTc (right) for iNPH and LIAS. Horizontal bars are the mean \pm standard deviation. The difference between the groups was statistically significant for RT $(p=0.014)$, but not for RTc $(p=0.61)$

clinical and monitoring parameters did not differ between centres in LIAS patients.

\section{Discussion}

This study investigated differences between the ICP profile of patients with iNPH and LIAS obtained from overnight recordings. We found mean ICP to be almost twice as high in LIAS patients compared to $\mathrm{iNPH}$; however, mean ICP in LIAS was still in a range of $\leq 15 \mathrm{mmHg}$, generally considered normal. This is consistent with the idea that despite the often complex CSF pressure versus flow relationship in hydrocephalus the chronic compensated nature of the aqueductal flow impairment and supratentorial CSF accumulation causes a non-critical elevation of ICP and explains higher values compared to an iNPH cohort where aqueductal CSF flow is not obstructed and free communication between inner and outer CSF spaces can be assumed.

Interestingly, iNPH patients showed a significant elevation in AMP and MWA as compared to LIAS, despite the fact that they had a lower ICP. This is a most important finding, since with a given exponential pressure-volume relationship higher ICP are to be associated with higher AMP/MWA, and not vice versa. The increased AMP/ MWA in iNPH at lower ICP indicates a very different pressure-volume relationship with the typical pressurevolume curve shifted to the left and a possibly narrower and steeper curve. This way the small intracranial volume changes caused by the cardiac pulsations induce larger ICP wave amplitudes at lower pressures.

Cerebrospinal reserve capacity and compliance measured with RAP were equally compromised in both groups, despite the higher average ICP values in LIAS. This again suggests a shift of the pressure-volume curve towards lower ICP values in iNPH, so that despite lower ICP a similar relationship between fluctuations in ICP and AMP results.

A previous investigation by González-Martínez et al. looked at differences between cerebrospinal fluid pressures, AMP and resistance to CSF outflow $\left(\mathrm{R}_{\text {out }}\right)$ derived from the lumbar compartment during lumbar infusion studies in NPH patients with open or stenotic cerebral aqueducts [14]. In their investigation baseline ICP and AMP did not differ between groups, but $R_{\text {out }}$ was higher in patients with an open aqueduct. This is again interesting as it indicates that the pressure differences between iNPH and LIAS patients obtained from the lumbar and cranial compartments might differ and should not be considered interchangeable.

Another possible explanation for the increased AMP/ MWA in iNPH is diminished dampening of the cardiac induced pulsations in the intracranial compartment. Previous studies have reported increased MWA in iNPH, but the causes for these increased pulsations remain elusive at this stage [15]. They can theoretically lie anywhere between the heart, where they originate and the ICP sensor, where they are measured. Vascular stiffening, changes in the viscoelastic properties of the aging brain, impaired CSF absorptive capacity for arterial pulsations and other CSF specific factors all need to be taken into consideration $[16,17]$. Whether the increase in AMP/MWA is a causative part of the pathophysiological disease process in iNPH or merely an associated epiphenomenon cannot be answered with this study. This study was also not designed to assess if increases in AMP/MWA can be used to select patients for VP-shunt surgery and may help differentiate shunt responders from non-responders. However, AMP/ MWA certainly deserves further attention in iNPH research.

RAP was increased in both groups, indicating similarly impaired reserve capacity in both types of chronic hydrocephalus. SLOW, measuring vasogenic ICP slow wave or b-wave activity was also not different between groups. No threshold values for SLOW in chronic hydrocephalus exist, but the observed average values of above $1.5 \mathrm{mmHg}$ can probably be considered abnormal [18].

The RT describes the time between the diastolic valley and systolic peak of the ICP pulse wave. It is thus, apart from intracranial compliance, dependant on the heart rate. RT was higher in iNPH, but the heart rate was similar between iNPH and LIAS. This might indicate that further analysis of the ICP pulse waveform morphology will be of interest in chronic hydrocephalus. This requires further sophisticated software analysis solutions, such as 
the MOCAIP algorithm, which are not available at our study centres [19].

This study has several limitations. ICP has been suggested to change with age and age has a confounding effect on our results. Older individuals likely have lower physiological ICP levels, but other factors such as vascular wall compliance are also changing with age. It is thus possible that the measured differences in ICP and AMP/MWA between the older iNPH and younger LIAS groups are mainly a function of age, and not disease. Our magnitude of ICP difference between iNPH and LIAS is however larger than a previous investigation measuring ICP in 'pseudonormal' patients across a wide range of age groups suggested. The data by Pedersen et al. found normal ICP to decrease by only $0.7 \mathrm{mmHg}$ per decade of life [20]. Our statistical models to investigate the influence of age on ICP and AMP had low adjusted $\mathrm{R}^{2}$, likely due to the small number of LIAS patients, and thus need to be interpreted with caution. Other parameters describing CSF dynamics, such as resistance to CSF outflow, also change with age and further research to understand the physiological evolution of ICP and AMP/MWA with age is necessary [21].

The small group of LIAS patients prohibited a more definite statistical analysis. Chronic LIAS accounted for only approximately $5 \%$ of all investigated adult CSF circulation disorders in both study centres. To increase the number of investigated LIAS patients to obtain a clearer statistical picture in this retrospective study was the main reason to combine data from two neurosurgical centres, both of which have regular experience with computerised ICP analysis of CSF circulation disorders using ICM + software. Whilst we were able to increase the number of analysed LIAS patients the combination of two centres might have also introduced bias into the analysis. For example, the proportion of iNPH and LIAS patients from both centres was different with a larger percentage of iNPH from Wollongong. In addition, ICP was higher in iNPH patients treated in Germany and lower in Australia. Whilst this might be incidental, it can also indicate bias in patient selection between hospitals or differences in clinical parameters that were not collected in this study, such as symptom duration or co-morbidities. Different ICP sensor equipment (Codman versus Raumedic) might have introduced further bias and should ideally be avoided in future prospective investigations.

\section{Conclusions}

ICP is non-critically elevated in LIAS and lower in iNPH. AMP/MWA as a marker of intracranial heartbeat related pulsations is elevated in iNPH. The origin of these increased pulsations necessitates further research to understand this possible mechanism of iNPH better. In both disease groups intracranial reserve capacity is impaired with a possible shift of the pressure-volume curve towards lower ICP values in iNPH. The physiological influence of age on ICP and derived parameters requires additional investigations.

\begin{abstract}
Abbreviations
iNPH: Idiopathic normal pressure hydrocephalus; VP-Shunt: Ventriculo-peritoneal shunt; CSF: Cerebrospinal fluid; LIAS: Late-onset idiopathic aqueductal stenosis of the adult; ICP: Intracranial pressure; MRI: Magnetic resonance imaging; CT: Computed tomography; ETV: Endoscopic third ventriculostomy; AMP: Intracranial pulse pressure amplitude calculated in the frequency domain; bpm: Beats per minute; RAP: Correlation coefficient (R) between amplitude (A) and pressure (P), a marker of cerebrospinal compensatory reserve capacity; HR: Heart rate; RESP: ICP amplitude of the respiration induced waves; RR: Respiratory rates; SLOW: Amplitude of the slow vasogenic waves of ICP; MWA: Intracranial pulse pressure mean wave amplitude calculated in the time domain; RT: Rise time of the ICP pulse pressure wave; RTc: Rise time coefficient of the ICP pulse pressure wave; $R_{\text {out }}$ : Resistance to CSF outflow.
\end{abstract}

\section{Acknowledgements}

We thankfully acknowledge the statistical advice of Joseph Descallar, University of New South Wales, South Western Sydney Clinical School, Liverpool, NSW, Australia.

The authors also thank Dr. Julian Zipfel, Department of Neurosurgery, Eberhard Karls University Hospital, Tübingen, Germany, for his help with providing ICM + data sets.

\section{Authors' contributions}

LMG planned the study, collected, analysed and interpreted patient data and critically revised the manuscript. TW collected patient data and critically revised the manuscript. MUS collected patient data and critically revised the manuscript. MJ conceptualised and planned the study, collected, analysed and interpreted patient data and drafted the manuscript. All authors read and approved the final manuscript.

Funding

This study did not receive specific funding.

Availability of data and materials

The data that support the findings of this study are available upon reasonable request from the corresponding author $[\mathrm{MJ}]$. The data are not publicly available due to them containing information that could compromise research participant privacy.

\section{Declarations}

\section{Ethics approval and consent to participate}

The local human research ethics committee approved this study (2019/ ETH1 1729) for the Australian patient cohort. Because of the retrospective nature of the study with analysis of routinely collected data the need for informed consent was waived. In Germany, retrospective analysis of data recorded as part of routine clinical management was appraised by the local ethics committee of Tübingen without the necessity of informed consent of patients examined in the past according to German Law ( $\$ 15$ Abs. 3 Landesdatenschutzgesetz).

\section{Consent for publication}

As per our ethics statement, this was a retrospective study, not requiring separate consent for publication. 


\section{Competing interests}

MJ has received funding from the Illawarra Health and Medical Research Institute for research on hydrocephalus related topics. He has received honoraria from B. Braun Australia Pty Ltd for presentations on hydrocephalus.

\section{Author details}

1 Department of Neurosurgery, Wollongong Hospital, Loftus Street, Wollongong, NSW 2500, Australia. ${ }^{2}$ Department of Neurosurgery, Eberhard Karls University Hospital, Tübingen, Germany. ${ }^{3}$ Illawarra Health and Medical Research Institute, Wollongong, NSW, Australia. ${ }^{4}$ University of New South Wales, South Western Sydney Clinical School, Liverpool, NSW, Australia. ${ }^{5}$ University of Wollongong, Wollongong, NSW, Australia.

Received: 6 February 2021 Accepted: 13 May 2021

Published online: 26 May 2021

\section{References}

1. Andren K, Wikkelsø C, Tisell M, Hellström P. Natural course of idiopathic normal pressure hydrocephalus. J Neurol Neurosurg Psychiatry. 2014;85:806-10.

2. Jaraj D, Wikkelsø C, Rabiei K, et al. Mortality and risk of dementia in normal-pressure hydrocephalus: a population study. Alzheimers Dement. 2017;13:850-7.

3. Jaraj D, Rabiei K, Marlow T, Jensen C, Skoog I, Wikkelsø C. Prevalence of idiopathic normal-pressure hydrocephalus. Neurology. 2014;82:1449-54

4. McAllister JP, Williams MA, Walker ML, Kestle JRW, Relkin NR, Anderson AM, et al. An update on research priorities in hydrocephalus: overview of the third national institutes of health-sponsored symposium "opportunities for hydrocephalus research: pathways to better outcomes." J Neurosurg. 2015;123:1427-38.

5. Fukuhara T, Luciano MG. Clinical features of late-onset idiopathic aqueductal stenosis. Surg Neurol. 2001;55:132-6.

6. Relkin N, Marmarou A, Klinge P, Bergsneider M, Black PM. .; Suppl. Diagnosing idiopathic normal-pressure hydrocephalus. Neurosurgery. 2005;57:S4-16.

7. Eide PK. Intracranial pressure parameters in idiopathic normal pressure hydrocephalus patients treated with ventriculo-peritoneal shunts. Acta Neurochir (Wien). 2006;148:21-9.

8. Evans WA. An encephalographic ratio for estimating ventricular enlargement and cerebral atrophy. Arch Neurol Psychiatry. 1942;47:931-7.

9. Smielewski P, Czosnyka M, Steiner L, Belestri M, Piechnik S, Pickard JD. $\mathrm{ICM}+$ : software for on-line analysis of bedside monitoring data after severe head trauma. Acta Neurochir (Wien). 2005;95 Suppl:43-9.
10. Czosnyka M, Guazzo E, Whitehouse M, Smielewski P, Czosnyka Z, Kirkpatrick $P$, et al. Significance of intracranial pressure waveform analysis after head injury. Acta Neurochir. 1996;138:531-41.

11. Kim DJ, Czosnyka Z, Keong N, Radolovich DK, Smielewski P, Sutcliffe MPF, et al. Index of cerebrospinal compensatory reserve in hydrocephalus. Neurosurgery. 2009;64:494-502.

12. Balestreri M, Czosnyka M, Steiner LA, Schmidt E, Smielewski P, Matta $B$, et al. Intracranial hypertension: what additional information can be derived from ICP waveform after head injury? Acta Neurochir (Wien). 2004; 146:131-41.

13. Eide PK. A new method for processing of continuous intracranial pressure signals. Med Eng Phys. 2006;28:579-87.

14. González-Martínez EL, Santamarta D. Does aqueductal stenosis influence the lumbar infusion test in normal-pressure hydrocephalus? Acta Neurochir (Wien). 2016;158:2305-10.

15. Eide PK, Sorteberg W. Diagnostic intracranial pressure monitoring and surgical management in idiopathic normal pressure hydrocephalus: a 6-year review of 214 patients. Neurosurgery. 2010;66:80-91.

16. Israelsson H, Carlberg B, Wikkelsø C, Laurell K, Kahlon B, Leijon G, et al. Vascular risk factors in INPH. Neurology. 2017;88:577-85.

17. Streitberger KJ, Wiener E, Hoffmann J, Freimann FB, Klatt D, Braun J, et al. In vivo viscoelastic properties of the brain in normal pressure hydrocephalus. NMR Biomed. 2010;24:385-92.

18. Czosnyka Z, Czosnyka M. Long-term monitoring of intracranial pressure in normal pressure hydrocephalus and other CSF disorders. Acta Neurochir (Wien). 2017;159:1979-80.

19. Hu X, Xu P, Scalzo F, Vespa P, Bergsneider M. Morphological clustering and analysis of continuous intracranial pressure. IEEE Trans Biomed Eng. 2009;56:696-705.

20. Pedersen SH, Lilja-Cyron A, Andresen M, Juhler M. The relationship between intracranial pressure and age-chasing age-related reference values. World Neurosurg. 2018;110:e119-23.

21. Albeck MJ, Skak C, Nielsen PR, Olsen KS, Børgesen SE, Gjerris F. Age dependency of resistance to cerebrospinal fluid outflow. J Neurosurg. 1998:89:275-8

\section{Publisher's Note}

Springer Nature remains neutral with regard to jurisdictional claims in published maps and institutional affiliations.
Ready to submit your research? Choose BMC and benefit from:

- fast, convenient online submission

- thorough peer review by experienced researchers in your field

- rapid publication on acceptance

- support for research data, including large and complex data types

- gold Open Access which fosters wider collaboration and increased citations

- maximum visibility for your research: over $100 \mathrm{M}$ website views per year

At $\mathrm{BMC}$, research is always in progress.

Learn more biomedcentral.com/submissions 